\title{
Massive hepatitis A outbreak in Sri Lanka in 2009: an indication of increasing susceptibility and epidemiological shift?
}

\author{
NJ Dahanayaka ${ }^{1}$, T Kiyohara ${ }^{2}$, SB Agampodi ${ }^{3}$ \\ Sri Lankan Journal of Infectious Diseases 2013 Vol.3(2):28-30 \\ DOI: http://dx.doi.org/10.4038/sljid.v3i2.5640
}

Key words: Hepatitis A, outbreak, Sri Lanka, endemic

\begin{abstract}
Sri Lanka experienced an unprecedented outbreak of hepatitis A in 2009. We extracted data from different sources to get a proper estimate of actual disease incidence during that period. This data show a total number of 13,477 cases in 2009 which is one of the largest outbreaks in recent history. We confirmed the etiological diagnosis as hepatitis A among armed forces towards the end of the outbreak. This massive outbreak shows the potential risk of future outbreaks due to epidemiological shifts of hepatitis A infection in Sri Lanka.
\end{abstract}

\section{Introduction}

In Sri Lanka, Hepatitis A (HA) occurs sporadically with a tendency for cyclic recurrence every 5-6 years (Figure 1). The average number of HA cases reported to the Sri Lanka Epidemiology Unit is around 3,000 cases per year with an estimated incidence of 15 cases per 100,000 population. Sri Lanka experienced an unprecedented outbreak of HA in 2009 which has not been described in the scientific literature. The objective of this paper is to report the extent and implications of the 2009 HA outbreak.

Figure 1: Disease surveillance data for Hepatitis A from $1990-2008$

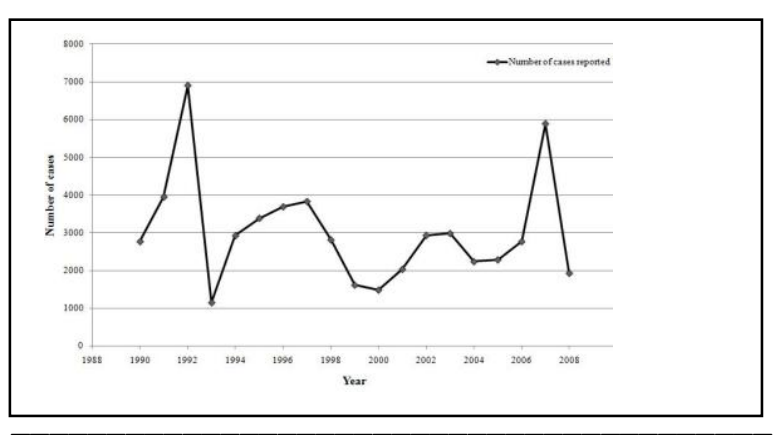

\section{Methods}

We retrieved published data from the Epidemiology Unit, Sri Lanka and unpublished data on reported cases of hepatitis A among the armed forces. These data from the armed forces were not included in the published national data due to the unavailability of a reporting district. Towards the end of the outbreak, we collected serum

\footnotetext{
${ }^{I}$ Department of Medicine, Faculty of Medicine and Allied Sciences, Saliyapura

${ }^{2}$ Department of Virology, National Institute of Infectious Diseases, Tokyo, Japan,

${ }^{3}$ Department of Community Medicine, Faculty of Medicine and Allied Sciences, Saliyapura
}

Address for correspondence: Dr Suneth Agampodi, Department of Community Medicine, Faculty of Medicine and Allied Sciences, Saliyapura, Anuradhapura. Email: sunethagampodi@yahoo.com, Tel: +94 (0)777880096 
samples from clinically suspected HA patients from the armed forces for disease confirmation.

Data reported from the Vavuniya district which was part of the conflict affected zone was analyzed. This data was obtained from the host population of the Vavuniya district (excluding IDPs), IDPs and armed forces. Hepatitis cases from the host populations of other districts in the Northern Province were obtained from published data.

\section{Results}

A total of 6855 cases of viral hepatitis were reported to the Epidemiology Unit by the $31^{\text {st }}$ of December 2009, of whom 5245 were from the Vavuniya district, including cases from the internally displaced persons (IDPs) camp. In addition, 6622 cases of hepatitis were recorded among armed forces operating in the Northern Province, resulting in a total number of 13,477 cases. Reported cases of HA from areas outside the North showed a very minor increase in numbers compared to the large outbreak in the Vavuniya district and among the armed forces (Figure 2). Of the 222 suspected patients from the armed forces tested for HA IgM antibodies towards the latter part of the epidemic, $214(97.27 \%)$ were positive.

Figure 2 - Epidemic curves of 2009 hepatitis A outbreak in Sri Lanka - disaggregated by conflict affected areas

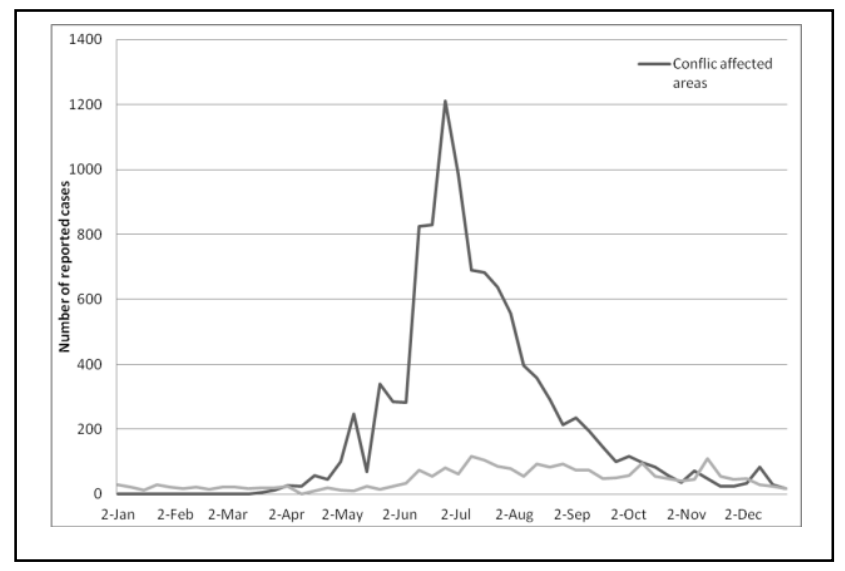

\section{Discussion and conclusion}

Our analysis confirmed the etiological diagnosis of the 2009 hepatitis outbreak among the armed forces. The similar spatial and temporal distribution of hepatitis cases in the Vavuniya district considered together with the clinical data provide sufficient epidemiological evidence to suggest that the total outbreak was due to HA. This is the largest reported outbreak of HA in Sri Lanka in the recent past. The disease incidence during 2009 was around 750 cases per 100,000 population. However,

this may be an underestimation of the actual disease incidence due to poor notification in routine surveillance, especially in emergency situations. As an example, the Communicable Diseases Updates prepared by the WHO Country Office was giving comparatively larger numbers of cases than what was reported to the Epidemiology Unit. ${ }^{1}$ The occurrence of this large outbreak questions the current understanding on HA epidemiology in Sri Lanka. Though WHO classifies Sri Lanka as a high endemic country ${ }^{2}$ and the Epidemiology Unit considers HA as endemic in Sri Lanka, ${ }^{3}$ massive common source outbreaks, as experienced in 2009, are not observed among adults in high endemic countries. This outbreak shows the high susceptibility of an adult population who escaped childhood infection, which is a feature of a low endemic country. ${ }^{4}$ However, the transitional economic and socio-demographic profile of Sri Lanka is different from other low endemic countries, which are mostly categorized as the developed world. Though the 2009 outbreak was contained in the Vavuniya district (IDP camps) and among the armed forces due to public health measures such as supply of sanitary water facilities, establishment of basic toilets and restriction of IDP 
movements during that time, the change in HA epidemiology poses a future risk of large HA outbreaks in Sri Lanka under unfavorable conditions. HA should be a priority condition in communicable disease control activities in all natural and manmade disasters where common source outbreaks are a possibility.

\section{References}

1. World Health Organization: Communicable Disease Weekly Updates. Available from http://www.whosrilanka.org/LinkFiles/Emergency_Health_Action_CD_News_No.-16.pdf

2. World Health Organization: Hepatitis A. Department of Communicable Disease Surveillance and Response. Geneva: World Health Organization; 2000: 6. Available at:

http://www.who.int/csr/disease/hepatitis/HepatitisA_whocdscsredc2000_7.pdf

3. Epidemiological Unit: Viral Hepatitis. In: Surveillance Case definitions for Notifiable Diseases in Sri Lanka. Colombo: Epidemiological Unit; 2010: 38.

4. Lemon SM: Type A viral hepatitis: epidemiology, diagnosis, and prevention. Clinical Chemistry 1997, 43:1494-99. Available at: http://www.clinchem.org/content/43/8/1494.full 